\title{
A Study of Constructing Menu Design Indices of B \& B in Taiwan
}

\author{
Yen-Cheng Chen ${ }^{1}$, Pei-Ling Tsui ${ }^{2,3^{*}}$, Hsin-I Chen ${ }^{1}$ and Shu-Hsin Ko ${ }^{1}$ \\ ${ }^{1}$ Department of Applied Science of Living, Chinese Cultural University, Taiwan \\ ${ }^{2}$ Department of Hospitality Management, National Taitung College, Taiwan \\ ${ }^{3}$ Graduate Institute of Technological and Vocational Education, National Taipei University of \\ Technology, Taiwan \\ *Corresponding author: j2217013@ms48.hinet.net
}

\begin{abstract}
In recent years with the rise of the sightseeing tour, the demand for a significant increase in tourist accommodation in Taiwan, thereby accelerating the thriving $B \& B$ industry. $B \& B$ with respect to the earlier operation, mostly from the house is the use of spare rooms and facilities in the $B \& B$ as a sideline business, and today's $B \& B$ industry in the face of fierce competition in the market $B \& B$, has been in the past to change the traditional distinctive Bed and Breakfast Bed and Breakfast offers including overall design, the experience on the ground ingredients, and offers specialty meals and meal service to enhance their competitiveness. Therefore, this study will focus on $B \& B$ Restaurant menu design evaluation indicators as a research subject, through the "fuzzy Delphi method (FDM)". The construction and analysis of restaurant menus and Breakfast assessment of index design to serve as $a B \&$ Band catering industry marketing manager reference management.
\end{abstract}

Keywords: Bed and breakfast, menu design, indicator

\section{INTRODUCTION}

As the prosperity of two-day weekend and tourism in Taiwan, the demand of accommodation has increased, which results in the development of the "bed and breakfast industry." However, instead of lowering the price to attract customers, the food specialty which every bed and breakfast provides has become one of the main attractions for tourists. Therefore, a lot of $b \& b$ s have dedicated themselves into enhancing the quality of every meal which leads to stimulating the potential of $b \& b$ industry. In the early phases of running $b \& b$, the owners turned their own residence into $a b \& b$ and used it as a sideline business. Nowadays, in order to face a more competitive market, those traditional houses have been transformed into a unique and one of a kind $\mathrm{b} \& \mathrm{~b}$ accommodations providing a very stylish living surrounding, local food, and good quality service. (Mills \& Thomas, 2008; Yang, Kimes \& Sessarego, 2009; Raab, Mayer \& Shoemaker, 2010; Mielby \& Frost, 2010; Ting, Pan \& Chou, 2010)

In the recent years, running restaurants and $b \& b$ has become a very popular issue. However, most of the research focuses on customer behavior and the overall management. Documents are mainly about marketing, customer satisfaction, and management (Wood \& Munoz, 2006). It's rare to see documents about the analysis of $b \& b$ menu. Nevertheless, the menu design can reflect the uniqueness and the diversity of a restaurant. The content of the menu can be adjusted based on different operation styles. Menu is one of the major factors of dominating a restaurant (Ninemeier, 2005; Kotschevar \& Withrow, 2008; Yang, Kimes \& Sessarego, 2009). B\&b not only provides distinguishing menu which can satisfy the need of customer market, but also brings out the glamour of local food by designing every dish, which can attract customers and customize every different need. Therefore, the design of the menu has become a crucial key to running a b\&b (Reynolds, Merritt \& Pinckney, 2005) .

According to the above documents, die to the fact that menu does influence every aspect of running a restaurant. Therefore, when designing a menu, the designer should consider the style of the restaurant, human resource, equipment, finance. The designer should also think about in what way and through what facility can create the unique style of the restaurant in order to satisfy the customers and meet the self quality management(Mill, 2007; Kotschevar \& With row, 2008;NinemeierA, 2005; Marshall, 2007).

As for the customers who are staying in the $b \& b$, menu is the medium which conveys their business concept to the customers. Almost every customer can get to know the local food, unique style, design concept by reading the menu. As for the owner of the $b \& b$ business, the content of the menu is related to the purchase of the ingredients, the preparation of the ingredients, and the service process. Besides from 


\section{Yen-Cheng Chen et al.}

the equipment of the $b \& b$, the food is one of the significant factors of running $a b \& b$. Food specialty is a main reason why customers want to stay for some nights. Not only $b \& b$ has to prepare the local food, but also has to get to know the customer preference. Also, when it comes to designing the menu, the owner of the $b \& b$ has to know what kind of equipment is needed when preparing every meal, how many workers they have, the workers technical level, and cost control.(Reynolds, Merritt \& Pinckney, 2005; Yang, Kimes \& Sessarego, 2009; Ting, Pan \& Chou, 2010)。

\section{METHOD}

The purpose of applying fuzzy Delphi method is to find suitable indicators of B \& B menu interior design. The fuzzy Delphi method is to utilize these indicators and prioritize them. The priorities of the indicators can provide a complete picture for decision makers of how will they prioritizing their menu efforts subject to limited resources (e.g., budget, time). Here we will provide a brief introduction of the methods we applied in this study

The process we implemented to collect, summarize and conclude consensus information is called fuzzy Delphi method. Theoretically, the Delphi process can be continuously iterated until consensus is determined to have been reached. However, literature pointed out that three iterations are often sufficient to collect the needed information and to reach a consensus in most cases (Brooks, 1979; Custer, Scarcella, \& Stewart, 1999; Cyphert \& Gant, 1971; Hsu \& Sandford, 2007; Ludwig, 1997). The following introduction, however, provides guidelines for up to four iterations in order to provide a more complete application guideline offuzzy Delphi method.

Round 1: In the first round, the Delphi process traditionally begins with an open-ended questionnaire. The open-ended questionnaire serves as the cornerstone of soliciting specific information about a content area from the Delphi subjects (Custer et al., 1999). In this study, for instance, this question would be to ask experts to provide all atmosphere dimensions and positive factors they could think of. After receiving subjects' responses, researchers need to convert the collected information into a well-structured questionnaire. This questionnaire is used as the survey instrument for the second round of data collection. It should be noted that it is both an acceptable and a common modification of the Delphi process format to use a structured questionnaire in Round 1 that is based upon an extensive review of the literature. The use of a modified Delphi process is appropriate if basic information concerning the target issue is available and usable (Kerlinger \& Lee, 2000).

Round 2: In the second round, each Delphi participant receives a second questionnaire and is asked to review the items summarized by the researchers based on the information collected in the first round. Accordingly, Delphi panelists may be required to rank orders of items to decide the priorities and preservations of them. In some cases, Delphi panelists are asked to state the rationale behind ranking priorities among items (Jacobs, 1996). In this round, consensus begins forming and the actual outcomes can be presented among the participants' responses (Jacobs, 1996).

Round 3: In the third round, each Delphi panelist receives a questionnaire that includes the items and ratings summarized by the researchers in the previous round and are asked to revise his/her judgments or to justify the reason he/she should remain outside of the consensus. This round gives Delphi panelists an opportunity to make further clarifications of both the information and their judgments of the relative

importance of the items. However, only a slight increase in the degree of consensus can be expected compared to the previous round (Jacobs, 1996; Weaver, 1971).

Round 4: In the fourth and often final round, researchers distribute the list of remaining items, their ratings, minority opinions, and items achieving consensus to the panelists. This round provides a final opportunity for participants to revise their judgments. It should be highlighted that the number of Delphi iterations depends largely on the degree of consensus sought by the investigators and can vary from three to five (Delbecq, Van de Ven, \& Gustafson, 1975).

\section{RESULT}

Based on the use of Delphi method, through four rounds of expert opinion collection, investigation and aggregation, this study summed up five important B \& B restaurant menu design evaluation indicators oriented to be able to be used to help attract B \& B customers a good impression. These five dimensions are: ingredients quality, local features, Decorative containers, food nutrition and delicious dishes, and 35 indicators, as shown in Table 1.

In the selection of indicators, the study asked the experts to delete the details of the indicators are too detailed, only that the general direction of the indicators. For example, the decorative containers in the dimensions of the 10 indicators, are clean, simple, distinguishing, homemade, pleasant, feels like home, 


\section{A Study of Constructing Menu Design Indices of B \& B in Taiwan}

bright, warm, health and safety, delicate. These 10 indicators do not specify which materials are used to match the material used to hold the breakfast, but rather to point out 10 different attributes that the customer may have varying degrees of preference.

Table1. B \& B Menu Design Dimensions and Factors

\begin{tabular}{|l|l|}
\hline Menu design dimension & Facets \\
\hline Ingredient quality & Fresh, no external flaw and no peculiar smell、 saturated and elastic, certificated \\
\hline Local features & $\begin{array}{l}\text { Local product, seasonal ingredient, local culture, creativity, unique ingredient, } \\
\text { based on local customs }\end{array}$ \\
\hline Decorative containers & $\begin{array}{l}\text { Clean, simple, distinguishing, homemade, pleasant, feels like home, bright, } \\
\text { warm, health and safety, delicate, transparent }\end{array}$ \\
\hline Food nutrition & $\begin{array}{l}\text { Caters to the customer need, healthy, calories label, contains dietary fiber, low in } \\
\text { sugar and salt, healthy cooking, less oil }\end{array}$ \\
\hline Delicious dish & $\begin{array}{l}\text { Flavorful smell, color-matched, proper serving amount, diversity in flavors, } \\
\text { unique and creative, tasty }\end{array}$ \\
\hline
\end{tabular}

In addition, after obtaining the defuzzification, the defuzzification 7 is used as the criterion for deletion. If the value of the ambiguity of the index is less than 7, it means that the index is not suitable for use as a restaurant menu. And therefore delete it. After deletion, this study made a total of 5 major dimensions, 31 indicators, please refer to Table 2 as a basis for assessment of the design of the B \& B restaurant menu.

Table2. Expert Fuzzy Delphi Method Results

\begin{tabular}{|c|c|c|c|c|}
\hline Dimension & Facets & Min & Max & Defuzzification \\
\hline \multirow{4}{*}{ Ingredient quality } & Fresh & 4 & 10 & 7.72 \\
\hline & no external flaw and no peculiar smell & 6 & 9 & 7.81 \\
\hline & saturated and elastic & 3 & 10 & 7.49 \\
\hline & certificated & 3 & 10 & 7.52 \\
\hline \multirow[t]{6}{*}{ Local features } & Local product & 5 & 10 & 8.25 \\
\hline & seasonal ingredient & 5 & 10 & 8.37 \\
\hline & local culture & 5 & 9 & 8.19 \\
\hline & unique ingredient & 2 & 10 & 7.48 \\
\hline & creativity & 3 & 10 & 7.88 \\
\hline & based on local customs & 3 & 10 & 7.92 \\
\hline \multirow[t]{12}{*}{ Decorative containers } & Clean & 5 & 10 & 7.58 \\
\hline & simple & 3 & 8 & 7.42 \\
\hline & distinguishing & 5 & 10 & 7.67 \\
\hline & bright & 9 & 9 & 7.71 \\
\hline & warm & 8 & 10 & 7.48 \\
\hline & health and safety & 3 & 8 & 7.74 \\
\hline & Homemade & 4 & 8 & 7.55 \\
\hline & pleasant & 3 & 8 & 7.31 \\
\hline & feels like home & 4 & 8 & 7.44 \\
\hline & delicate & 2 & 5 & 4.16* \\
\hline & transparent & 2 & 7 & 6.10* \\
\hline & feels like home & 4 & 8 & 7.50 \\
\hline \multirow[t]{7}{*}{ Food nutrition } & Caters to the customer need & 8 & 9 & 7.49 \\
\hline & healthy & 8 & 10 & 7.35 \\
\hline & calories label & 7 & 9 & 7.78 \\
\hline & contains dietary fiber & 3 & 10 & 7.30 \\
\hline & low in sugar and salt & 3 & 10 & 7.40 \\
\hline & less oil & 4 & 8 & 7.38 \\
\hline & healthy cooking & 7 & 10 & 7.52 \\
\hline \multirow[t]{6}{*}{ Delicious dish } & Flavorful smell & 7 & 10 & 7.55 \\
\hline & color-matched & 2 & 9 & 7.42 \\
\hline & proper serving amount & 8 & 10 & 7.56 \\
\hline & unique and creative & 2 & 7 & $6.48 *$ \\
\hline & tasty & 2 & 5 & $5.52 *$ \\
\hline & diversity in flavors & 4 & 7 & 7.23 \\
\hline
\end{tabular}

\footnotetext{
"Inappropriate metrics defuzzification less than 7, to delete
} 


\section{CONCLUSION}

This study uses the fuzzy Delphi method to gather a group of experts from related fields to comprehend the five dimensions and 31 indexes of B \& B breakfast design, which is of great contribution to the development of academic theory and the application of B \& B catering industry. Among them, in academic theory, the 31 indicators proposed in this study can be used as the basis for the development of the future academic research on the design and evaluation of the B \& B breakfast. In addition, in practice-oriented applications, the B \& B operators can refer to the five dimensions of this study, 31 indicators, scientific rigorous and accurate way, during breakfast preparation and brand marketing can make more accurate strategic marketing, But also as a B \& B breakfast design assessment tools to design a unique style and characteristics of the breakfast.

From the previous research, there are three aspects in the competitive market of catering industry: food, style, atmosphere(Auty, 1992). However, improving the taste and the quality of the food is the goal every business manager wants to achieve, it takes a big amount of time to practice. Plus, it is only possible by constantly stimulating the creativity. As for those who wants to be outstanding, focusing on developing the uniqueness of the breakfast is one of the best ways to require higher profit.

Lastly, although this research uses Delphi method based on experts opinions and collections, they are still the minority of people. Therefore, the index from this research still needs to be tested as well as broaden the scope of the sample in order to understand the ability of generalization. This research is aimed to contribute more to this area.

\section{REFERENCES}

Kotschevar, L. H., \& Withrow, D. (2008). Management by menu. New Jersey: WILEY.

Marshall, A. (2007) Take Unforeseen Risks in Restaurant Service off the Menu. Hotel and Motel Management, 222(19), 8.

Mielby, L. H., \& Frost, M. B. (2010). Expectations and Surprise in a Molecular Gastronomic Meal. Food Quality and Preference, 21(2), 213-224.

Mill, R. C. (2007). Restaurant Management. Customers, Operations, and Employees (3rd Ed.). New Jersey: Pearson Prentice Hall.

Mills, J. E., \& Thomas, L. (2008). Assessing Customer Expectations of Information Provided on Restaurant Menus: a Confirmatory Factor Analysis Approach. Journal of Hospitality \& Tourism Research, 32(1), 62-88.

Ninemeier, J. D. (2005). Management of Food and Beverage Operations. New York: Educational Institute of the AHMA.

Raab, C., Mayer, K., \& Shoemaker, S. (2010). Menu Engineering Using Activity-Based Costing: an Exploratory Study Using a Profit Factor Comparison Approach. Journal of Hospitality \& Tourism Research, 34(2), 204-224.

Reynolds, D., Merritt, E. A., \& Pinckney, S. (2005). Understanding Menu Psychology: an Empirical Investigation of Menu Design and Consumer Response. International Journal of Hospitality and Tourism Administration, 6(1), 1-10.

Stutt, A. T., and Wortman, J. F., (2005). Hotel and lodging management: An introduction.New York: John Wiley \& Sons, Inc.

Ting, P. H., Pan, S., \& Chou S. S. (2010). Finding Ideal Menu Items Assortments: An Empirical Application of Market Basket Analysis. Cornell Hospitality Quarterly, 51(4), 492-501.

Wood, N. T., \& Munoz, C. L. (2006). 'No Rules, Just Right' or is It? The Role of Themed Restaurants as Cultural Ambassadors. Tourism and Hospitality Research, 7(3/4), 242-255.

Yang, S. B., Kimes, S. E., \& Sessarego, M. M. (2009). Menu Price Presentation Influences on Consumer Purchase Behavior in Restaurants. International Journal of Hospitality Management, 28(1), 157-160. 